\title{
Backcrossing to different parents produced two distinct hybrid species
}

\author{
Donglei Wang ${ }^{1}$, Ji Wang ${ }^{1}$, Hao $\mathrm{Bi}^{1}$, Jianquan $\mathrm{Liu}^{1}$, and Dafu $\mathrm{Ru}^{2}$ \\ ${ }^{1}$ Sichuan University \\ ${ }^{2}$ Lanzhou University
}

October 19, 2020

\begin{abstract}
Repeated homoploid hybrid speciation (HHS) events with the same parental species have rarely been reported. In this study, we used population transcriptome data to test paraphyly and HHS events in one conifer Picea brachytyla. All analyses identified and supported non-sister relationships for the two lineages of $P$. brachytyla. The southern lineage was placed within the recircumscribed $P$. likiangensis species complex (PLSC) while P. brachytyla sensu stricto (s.s.), comprising only the northern lineage, parallels both PLSC and the closely related $P$. wilsonii. In addition, both phylogenetic and coalescent analyses suggested that P. brachytyla s.s. arose from homoploid hybrid speciation between the ancestor of the PLSC before its diversification (into the current varieties or species), and $P$. wilsonii, through an intermediate hybrid lineage at an early stage and backcrossing to the ancestral PLSC. These two parental ancestors also produced another homoploid hybrid species, P. purpurea, in the same way but at a later stage, through the same extinct lineage but backcrossing to the other parent, $P$. wilsonii. We reveal the first case that backcrossing to different parents of the same extinct hybrid lineage produced two different hybrid species. Our results highlight the existence of more reticulate evolution during species diversification in the spruce genus and more complex homoploid hybrid events than have previously been identified.
\end{abstract}

\section{Hosted file}

Picea brachytyla_2020-10-17-MolecularEcology.pdf available at https://authorea.com/users/ 368214/articles/487427-backcrossing-to-different-parents-produced-two-distinct-hybridspecies 